\title{
Auswirkungen des Erwachsenenschutzgesetzes auf die Behandlung urteilsunfähiger Patienten
}

Die nachfolgenden Ausführungen zu den Konsequenzen für die psychiatrische Behandlung erscheinen als zweiter Teil des Aufsatzes zu den Neuerungen im Erwachsenenschutzgesetz. Im ersten Teil, der in der letzten Ausgabe der SÄZ erschienen ist, wurde die somatische Behandlung erläutert.

\section{Ursina Pally Hofmann}

Dr. iur., Rechtsanwältin, Stv. Leiterin Rechtsdienst

Korrespondenz: Dr. iur. Ursina Pally Hofmann FMH Rechtsdienst Elfenstrasse 18 CH-3000 Bern 15 ursina.pally[at]fmh.ch

\section{Psychiatrische Behandlung: \\ 1. Fürsorgerische Unterbringung (FU)}

Leidet eine Patientin an einer psychischen Störung oder an geistiger Behinderung, ist sie schwer verwahrlost, darf sie in einer geeigneten Einrichtung untergebracht werden, wenn die nötige Behandlung oder Betreuung nicht anders erfolgen kann. Beim Entscheid werden die Belastung und der Schutz von Angehörigen und Dritten mitberücksichtigt. Sobald die Voraussetzungen für die Unterbringung nicht mehr erfüllt sind, ist die betroffene Person zu entlassen. Sowohl die betroffene Person selbst als auch ihr nahestehende Personen können jederzeit um Entlassung ersuchen [1]. Die Einweisung eines Patienten darf nicht durch seine Angehörigen veranlasst werden. Eine diesbezügliche Vertretungsberechtigung fehlt. Die Einweisung ist grundsätzlich durch die Erwachsenenschutzbehörde zu veranlassen, die Kantone können Ärzte im Rahmen einer ausserordentlichen Kompetenz ebenfalls dazu ermächtigen. Die von einem Arzt festgelegte Unterbringung darf allerdings höchstens sechs Wochen betragen. Er hat den Patienten selbst zu untersuchen. Der Entscheid des Arztes ist schriftlich festzuhalten, zu begründen, der betroffenen Person sofort zu eröffnen und mit einer Rechtsmittelbelehrung zu versehen. Zudem ist - sofern möglich - eine nahestehende Person über die Unterbringung und die Möglichkeit, den Entscheid bei einem Gericht anzufechten, zu informieren [2].

\subsection{Patientenverfügung, Angehörige} und Vertrauensperson

Eine Patientenverfügung ist bei der stationären Behandlung von urteilsunfähigen volljährigen Patienten im Rahmen einer Fürsorgerischen Unterbringung, und wenn es nur um die Behandlung von psychischen Beschwerden geht, nicht verbindlich, sondern nur $z u$ berücksichtigen [3]. Ein Abweichen davon sollte aber mit Zurückhaltung erfolgen und muss begründet werden [4].

Im Gegensatz zur Behandlung von somatischen Erkrankungen haben die Angehörigen und Nahestehenden kein Vertretungsrecht im Rahmen einer psychiatrischen Behandlung.
Die untergebrachte Person kann aber eine urteilsfähige Vertrauensperson bestimmen, die sie während des Aufenthaltes und in allfälligen Verfahren unterstützt, sofern sie sich nicht freiwillig in der Einrichtung aufhält. Die Vertrauensperson kann Einsicht in die Krankengeschichte und die Akten nehmen, sofern sie über eine entsprechende Vollmacht verfügt. Die Vertrauensperson soll die untergebrachte Person über ihre Rechte aufklären und sie bei deren Wahrnehmung unterstützen, bei Konflikten vermitteln und der betroffenen Person dabei behilflich sein, die Kontakte mit der Aussenwelt aufrechtzuerhalten [5].

\subsection{Rolle der Ärztin}

Die behandelnde Ärztin hat unter Beizug der betroffenen Person sowie allenfalls der Vertrauensperson einen schriftlichen Behandlungsplan zu erstellen. Sowohl die untergebrachte als auch die Vertrauensperson sind über die geplanten medizinischen Massnahmen aufzuklären. Die urteilsfähige betroffene Person hat dem Behandlungsplan zuzustimmen, wobei auch eine mutmassliche Zustimmung genügt. Keinesfalls darf die Zustimmung von einer anderen

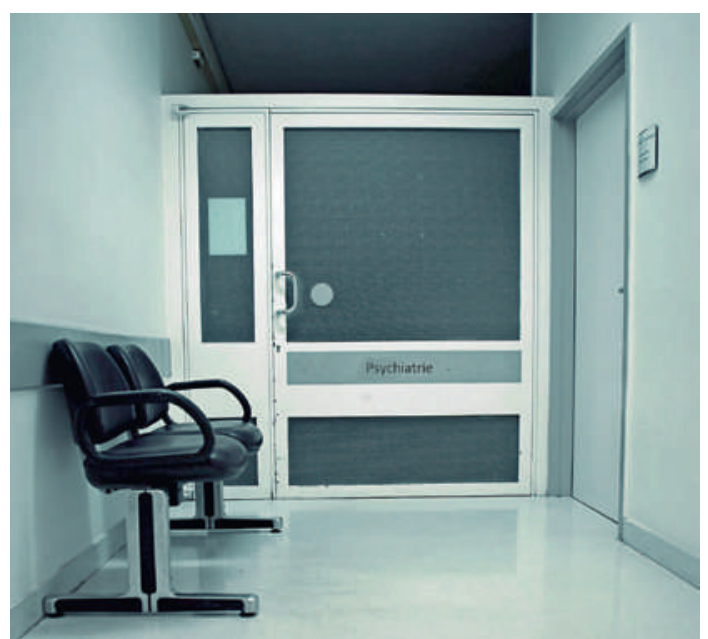

Eine Einweisung im Rahmen der Fürsorgerischen Unterbringung ist grundsätzlich durch die Erwachsenenschutzbehörde zu veranlassen. 
Person eingeholt werden [6]. Ist die betroffene Person nicht in der Lage, dem Behandlungsplan zuzustimmen, ist ihre Patientenverfügung zu berücksichtigen. Gibt es keine Hinweise auf den mutmasslichen Patientenwillen oder kann dieser nicht berücksichtigt werden, ist von der fehlenden Zustimmung der betroffenen Person auszugehen. Der Chefarzt der Abteilung kann die vom behandelnden Arzt im erstellten Behandlungsplan vorgesehenen medizinischen Massnahmen schriftlich anordnen, wenn die folgenden Voraussetzungen gegeben sind: Ohne Behandlung droht ein ernsthafter gesundheitlicher Schaden oder Dritte sind ernsthaft gefährdet; die betroffene Person ist betreffend ihre Behandlungsbedürftigkeit urteilsunfähig; es steht keine weniger einschneidende Massnahme zur Verfügung. Die Anordnung muss der betroffenen Person sowie der Vertrauensperson schriftlich mitgeteilt werden, und es ist eine Rechtsmittelbelehrung anzufügen. Die betroffene Person kann das Gericht anrufen [7].

In Notfallsituationen können unerlässliche Massnahmen zum Schutz der betroffenen Person oder Dritter durch das Dienst habende ärztliche medizinische Personal sofort und ohne Erstellen eines Behandlungsplans ergriffen werden. Falls der Wille der Person, die behandelt werden soll, bekannt ist, ist dieser zu berücksichtigen [8].

\section{Angehörige und Nahestehende haben kein Vertretungs- recht im Rahmen einer psychiatrischen Behandlung.}

Die Voraussetzungen für die Durchführung von Massnahmen zur Einschränkung der Bewegungsfreiheit sind dieselben wie in Wohn- oder Pflegeeinrichtungen. Im Rahmen einer FU ausgesprochene bewegungseinschränkende Massnahmen können nach schriftlicher Anrufung eines Gerichts entsprechend überprüft werden [9].

\section{Freiwilliger stationärer Aufenthalt}

Hält sich eine Person mit psychischer Störung freiwillig in einer Einrichtung auf und will sie diese wieder verlassen, kann die ärztliche Leitung sie für längstens drei Tage zurückbehalten. Vorausgesetzt ist, dass die Person sich selbst an Leib und Leben oder das Leben oder die Gesundheit Dritter gefährdet. Die zurückbehaltene Person wird mittels schriftlicher Anordnung darüber informiert, dass sie ein Gericht anrufen kann. Liegt kein Entscheid betreffend die weitere Unterbringung vor, darf die Einrichtung nach drei Tagen verlassen werden. Es ist davon auszugehen, dass während dieser Zurückbehaltung keine Behandlung gegen den Willen der betroffenen Person durchgeführt werden darf, es sei denn, es handle sich um die Vermeidung einer akuten Selbstgefährdung [10].

\section{Austrittsgespräch und Nachbetreuung}

Wenn eine Rückfallgefahr besteht, soll die behandelnde Ärztin mit der betroffenen Person möglichst vor der Entlassung für den Fall einer erneuten Unterbringung die Behandlungsgrundsätze vereinbaren. Diese sind zu dokumentieren. Diese Massnahme soll dazu dienen, die Wünsche und Vorstellungen des Patienten für einen allfälligen weiteren Aufenthalt festzuhalten und die Behandlung zu optimieren [11].

Die Kantone sind verpflichtet, die Nachbetreuung zu regeln. Dabei geht es nicht um die medizinische Behandlung, sondern freiwillige Angebote,die den Betroffenen und ihren Angehörigen neben den behördlichen Massnahmen, wie den Beistandschaften, zur Verfügung stehen.

Daneben können die Kantone ambulante Massnahmen vorsehen, die entweder freiwillig in Anspruch genommen oder zwangsweise durchgeführt werden können. Die Anordnung der Erwachsenenschutzbehörde, sich nach der Entlassung einer medikamentösen Behandlung zu unterziehen, gilt als Zwangsbehandlung, wenn der Patient damit rechnen muss, erneut in eine Klinik eingewiesen zu werden, sofern er sich der Anordnung widersetzt. Die Zwangsbehandlung gilt als schwerer Eingriff in die körperliche und geistige Unversehrtheit und der Menschenwürde [12]. Die Kantone haben verschiedene Massnahmen, wie z.B. Beratung, Therapie, ärztliche Behandlung, Medikamenteneinnahme, Verhaltensanweisungen, Kontrollen, Bestimmungen über den Aufenthalt vorgesehen. In einzelnen Kantonen ist die zwangsweise Durchführung dieser Massnahmen vorgesehen, andere Kantone sehen keine zwangsweise Durchführung vor.

In der nächsten Ausgabe der SÄZ wird über erste Erfahrungen in der Praxis berichtet.

\section{Referenzen}

1 Art. 426 ZGB.

2 Art. 428 ff. ZGB.

3 Muss im Rahmen einer FU ein somatisches Leiden behandelt werden, ist die Patientenverfügung verbindlich.

4 Art. 433 Abs. 3 ZGB; BSK-Erw.Schutz-Geiser/Etzensberger, N 15 f. zu Art. 433 ZGB.

5 Art. 432 ZGB.

6 Art. 433 ZGB

7 Art. 434, 439 ZGB.

8 Art. $435 \mathrm{ZGB}$.

9 Art. 438 f. ZGB.

10 Art. 427 ZGB; BSK-Erw.Schutz-Geiser/Etzensberger, N 8 ff.

11 Art. 436 ZGB.

12 Art. 437 ZGB; Urteil des Bundesgerichts (5A_666/2013) vom 7. Oktober 2013 E. 3. 